\title{
Prevenção e reabilitação de desordens músculo-esqueléticas relacionadas ao trabalho: uma visão integrada para promoção de saúde ocupacional
}

\author{
Prevention and rehabilitation of work-related musculoskeletal \\ disorders: an integrated view for occupational health promotion
}

\author{
Edgar Ramos Vieira ${ }^{1}$ \\ 'Pós-Doutorado em Metodologia Qualitativa pela International Institute for Qualitative Methodology, Canadá; Doutor em Ciência \\ da Reabilitação pela University of Alberta, Canadá; Professor Assistente do Departamento de Fisioterapia, Florida International \\ University - FIU. Miami, FL - USA.
}

Endereço para correspondência

Edgar Ramos Vieira

Florida International University, 11200 SW 8th St.

Florida International U
MM campus, AHC3

Miami, FL - 33199 - USA

EdgarRamosVieira@gmail.com

\begin{abstract}
Resumo
Dentre os objetivos principais da ergonomia e da fisioterapia do trabalho estão a prevenção e a reabilitação dos distúrbios musculoesqueléticos relacionadas ao trabalho. Estas afecções, conhecidas como LER/DORT, representam, no Brasil, um dos problemas de saúde mais comuns relacionados ao trabalho. Este artigo discute e ressalta a relação entre a ergonomia e a fisioterapia do trabalho para evitar a segregação e fragmentação destas áreas de trabalho complementares. Um modelo integrado de prevenção e reabilitação de LER/DORT é discutido e esquematicamente apresentado. Os profissionais envolvidos na prevenção de lesões ocupacionais devem encarar a reabilitação das LER/DORT como prevenção de incapacidade para o trabalho - prevenção secundária. Enquanto que os profissionais da reabilitação devem encarar a prevenção primária das LER/DORT como reabilitação com enfoque no ambiente e sistema de trabalho ao invés de reabilitação com enfoque no paciente/trabalhador. Acredita-se que esta conduta resultará em maior sucesso no gerenciamento das LER/DORT que atualmente afetam tantos trabalhadores e sobrecarregam o sistema previdenciário.
\end{abstract}

Descritores: Engenharia humana; Prevenção primária; Prevenção secundária; Reabilitação; Transtornos traumáticos cumulativos.

\begin{abstract}
The prevention and rehabilitation of work-related musculoskeletal disorders are among the main goals of ergonomics and work physical therapy. These disorders are known in Brazil as LER/DORT. They represent one of the most common work-related health problem. This article discusses and highlights the relationship between ergonomics and work physical therapy to avoid the segregation and fragmentation of these complementary areas of work. An integrated model of prevention and rehabilitation of LER/DORT is discussed and schematically presented. The professionals involved in the prevention of occupational injuries should view LER/DORT rehab as work disability prevention - secondary prevention. While the rehab professionals should face primary prevention of LER/ DORT as rehabilitation with focus on the work environment and work system as opposed to rehab focusing on the patient/worker. This integration may result in bigger success in managing LER/DORT, which currently affects many workers and strongly impact on the workers' compensation system.
\end{abstract}

Key words: Cumulative trauma disorders; Human engineering; Primary prevention; Rehabilitation; Secondary prevention. 


\section{Introdução}

O Programa Mundial de Ação da Organização Mundial de Saúde definiu há vinte anos e reafirmou em 2001 que a prevenção, a reabilitação e a igualdade de oportunidade para os deficientes físicos são seus principais objetivos ${ }^{1}$. A ergonomia e a fisioterapia do trabalho são instrumentais para atingir esses objetivos porque, dos objetivos principais de ambas as áreas, estão a prevenção e reabilitação dos distúrbios músculoesqueléticos relacionadas ao trabalho. No Brasil, estes distúrbios são denominados lesões por esforços repetitivos (LER) ou distúrbios osteomusculares relacionados ao trabalho (DORT). Genericamente estas afecções são conhecidas como LER/DORT. Dentre estas estão as lombalgias ocupacionais, as tendinites no ombro e punho, e a síndrome do túnel do carpo quando são relacionadas ao trabalho².

As LER/DORT representam, no Brasil, um dos problemas de saúde mais comuns relacionados ao trabalho, e sua frequência, causa e/ou severidade é modificada pelo trabalho ${ }^{3}$. Para dar uma idéia da dimensão do problema, em 2002 um total de 105.514 doenças relacionadas ao trabalho foi registrado no Brasil pelo Instituto Nacional de Seguro Social (INSS). Contudo, estes dados não representam a real incidência de doenças relacionadas ao trabalho, pois esta informação inclui apenas trabalhadores assegurados pelo Seguro de Acidentes de Trabalho (SAT), do INSS. De acordo com o Instituto Brasileiro de Geografia e Estatística (IBGE), 75.471 .556 brasileiros estavam empregados em 2001, sendo que, destes, apenas 22.903.311 estavam assegurados pelo SAT. Portanto, apenas um terço da população de trabalhadores foi incluído nos casos registrados de doenças relacionadas ao trabalho. Dessa forma, o número real de casos é muito maior do que o apresentado. De acordo com uma pesquisa realizada na cidade de São Paulo, que incluiu 1.072 trabalhadores, estimou-se que 310.000 trabalhadores $(6,6 \%$ da população) tiveram LER/DORT em 2001, apenas na capital paulista ${ }^{4}$.

Ergonomistas e profissionais da área de saúde ocupacional precisam trabalhar juntos para ter um impacto sobre os problemas relacionados ao trabalho ${ }^{5}$. Este artigo discute e ressalta a relação entre a ergonomia e a fisioterapia do trabalho para evitar a segregação e fragmentação dessas áreas de trabalho complementares. Um modelo integrado de prevenção e reabilitação de LER/DORT é sugerido, discutido e esquematicamente apresentado.

\section{Métodos}

Uma revisão da literatura sobre ergonomia da reabilitação e fisioterapia do trabalho foi realizada. Artigos contendo os termos "occupational physical therapy", "work physical therapy" e "rehabilitation ergonomics" foram pesquisados nas bases de dados Science Direct, Pubmed, Scirus e Web of Science. Adicionalmente, os websites da Associação Brasileira de Ergonomia (ABERGO), da Associação Internacional de Ergonomia (IEA), da Associação de Ergonomistas Canadenses (ACE), da Associação Americana de Fisioterapia (APTA), da Associação Canadense de Fisioterapia (CPA), da Sociedade Brasileira de Fisioterapia do Trabalho (SOBRAFIT), da Associação Nacional de Fisioterapia do Trabalho (ANAFIT) e de diversas agências de compensação dos trabalhadores foram pesquisados para obter as informações e fundamentar as discussões apresentadas neste artigo.

\section{Resultados}

As informações relevantes para o presente artigo disponíveis na literatura e nos websites citados foram divididas, apresentadas e discutidas nos seguintes tópicos: ergonomia da reabilitação, fisioterapia do trabalho, e a filosofia comum aos ergonomistas e fisioterapeutas do trabalho.

\section{Ergonomia da reabilitação}

De acordo com a Associação Internacional de Ergonomia (IEA: http://www.iea.cc), ergonomia é a ciência que estuda a relação entre o ho- 
mem e o trabalho. Seus objetivos são modificar os sistemas de trabalho para melhor adequá-lo às características, capacidades, e limitações dos trabalhadores, e maximizar o desempenho no trabalho otimizando a funcionalidade sem comprometer o conforto e segurança dos trabalhadores. A Associação Brasileira de Ergonomia (ABERGO: http://www.abergo.org.br) foi criada em 1983. No artigo "In praise of Brazilian ergonomics", Wisner ${ }^{6}$ afirma que "os ergonomistas Brasileiros corretamente preocupam-se com a saúde dos trabalhadores" (p. 417). De acordo com O'Neill5, "nos países em desenvolvimento industrial, a ênfase precisa ser na prevenção e deveria ser uma prioridade de desenvolvimento nacional" (p. 636). O mesmo autor ressalta que as principais preocupações ergonômicas nesses países em desenvolvimento são a carga física de trabalho, as condições ambientais, o design dos equipamentos e a saúde e segurança dos trabalhadores. Contudo, uma revisão dos estudos sobre intervenções ergonômicas na América Latina, durante os últimos 20 anos, demonstrou que as intervenções mais recentes têm utilizado uma metodologia mais participativa e que os aspectos organizacionais do trabalho têm recebido crescente atenção ${ }^{7}$.

Em 1978, o Dr. Kumar da Universidade de Alberta no Canadá, propôs a denominação "ergonomia da reabilitação" para a área de interação entre as disciplinas de reabilitação e ergonomia $^{8}$. Em 1993, a IEA formou um comitê científico para esta área. Sequencialmente, em 1994, houve o Simpósio Internacional de Ergonomia da Reabilitação, em Toronto, no Canadá, como parte do XII Congresso Internacional da IEA 9 . Atualmente, a ABERGO possui o grupo técnico em prevenção de lesões músculoesqueléticas / reabilitação que tem como finalidade reunir profissionais de diferentes áreas com o propósito de atuar buscando soluções para o quesito prevenção de LER/DORT (http://www.simucad.dep. ufscar.br/projetos/gt_abergo/). Este grupo técnico é coordenado e possui vários membros fisioterapeutas. A prática da ergonomia da reabilitação requer conhecimentos em anatomia, antropome- tria, fisiologia e biomecânica. Todas estas disciplinas são partes do conteúdo curricular dos cursos de graduação em fisioterapia. Portanto, estes profissionais possuem os requisitos básicos para que após uma especialização possam trabalhar na área de ergonomia da reabilitação.

\section{Fisioterapia do Trabalho}

É necessário que o processo de reabilitação do trabalhador lesado seja holístico, considerando a necessidade de independência econômica através da capacidade física para trabalhar ${ }^{10}$. A fisioterapia tradicional concentra-se principalmente no restabelecimento da funcionalidade ao nível das atividades de vida diária. Por outro lado, a fisioterapia do trabalho precisa ser mais completa tendo que restabelecer a funcionalidade ao nível necessário para a realização da atividade ocupacional do trabalhador lesado e prevenir lesões e re-lesões relacionadas ao trabalho. A fisioterapia do trabalho engloba a reabilitação e prevenção secundária, mas também incorpora o delineamento e implementação de programas de prevenção primária. Os programas de prevenção deveriam incluir avaliações da atividade ocupacional e intervenções, não focando apenas na ginástica laboral (uma tendência frequentemente observada atualmente no Brasil). Mesmo porque os efeitos de alongamentos na prevenção de LER/ DORT são contraditórios ${ }^{11}$.

Os Estados Unidos não possuem uma associação de fisioterapia do trabalho. Contudo, a Sessão Ortopédica da Associação Americana de Fisioterapia (APTA) possui um grupo de interesse em "fisioterapia ocupacional" cujo website é http://www.orthopt.org/sigs/occupational_health_sig/. De acordo com a APTA, o papel do fisioterapeuta na saúde ocupacional inclui o exame e avaliação de indivíduos com fator(es) de risco relacionados ao trabalho, deficiências, limitações funcionais, incapacidades, ou outras condições relacionadas à saúde, as quais previnam o indivíduo de realizar suas atividades ocupacionais, com o objetivo de determinar um diagnóstico, um prognóstico, e de implementar 
intervenções conforme à necessidade ${ }^{12}$. A APTA publicou guias para a prática fisioterápicas em diferentes áreas, inclusive para fisioterapeutas atuando na área de prevenção de lesões ocupacionais/ergonomia ${ }^{13}$.

De acordo com a Associação Canadense de Fisioterapia (CPA), cujo website é www.physiotherapy.ca, os fisioterapeutas requerem uma licença profissional do colégio de sua província para atuar profissionalmente no Canadá. O colégio de fisioterapeutas de cada província é a entidade que licencia e regula a profissão, sendo responsável por garantir o interesse público em relação aos serviços fisioterápicos prestados. Dessa forma, a função principal dos colégios é de proteger o paciente/público, enquanto que o papel secundário é de regular e direcionar a profissão. Cada colégio é responsável por garantir que os fisioterapeutas, atuando na província, estejam licenciados e tenham atingido os requisitos mínimos que cada província define (frequentemente uma prova com nota mínima preestabelecida), para poder atuar profissionalmente, independentemente de sua especialidade ou área de trabalho.

Existem algumas organizações no Canadá que fazem a reabilitação ocupacional tais como as agências de compensação dos trabalhadores de Alberta (www.wcb.ab.ca) e da Colômbia Britânica (www.worksafebc.com), e a Agência de Segurança e Seguro do Trabalhador de Ontário (www.wsib.ca). Estas agências funcionam como seguradoras e são financiadas diretamente pelas indústrias e empregadores. Além destas agências, existem empresas privadas especializadas na avaliação dos trabalhadores lesados tais como o Centro de Avaliação de Disfunções (DAC) (www.disability-assessment.com).

Embora a prevenção faça parte da educação do fisioterapeuta desde 1983 (Resolução N.4, CFE), foi durante o fim dos anos 80 e início dos anos 90 que os fisioterapeutas brasileiros começaram a participar de programas de prevenção de lesões relacionadas ao trabalho ${ }^{14}$. Apenas mais recentemente é que houve um maior desenvolvimento na área de fisioterapia do trabalho no Brasil. Em 2004, houve dois grandes eventos nesta área no país: o Congresso Internacional de Fisioterapia do Trabalho da Sociedade Brasileira de Fisioterapia do Trabalho (SOBRAFIT), e o I Congresso Internacional de Fisioterapia do Trabalho promovido pela Associação Nacional de Fisioterapia do Trabalho (ANAFIT). As duas organizações profissionais (SOBRAFIT - criada em 2002 e ANAFIT - criada em 1998) possuem funções similares e, portanto, foram integradas formando a Associação Brasileira de Fisioterapia do Trabalho (ABRAFIT). O papel da ABRAFIT deveria ser de licenciar e regular a prática profissional dos fisioterapeutas atuando na área ocupacional na reabilitação de trabalhadores lesados e em intervenções para prevenir LER/DORT (incluindo análises das demandas físicas do trabalho, intervenções ergonômicas, recomendações e programas de treinamento dos trabalhadores). Além disso, a associação deverá representar os interesses dos "fisioterapeutas do trabalho" diante das agências reguladoras tais como o ministério do trabalho. Por fim, a agência deverá promover educação continuada na área incluindo seminários, workshops, simpósios, e congressos.

\section{A filosofia em comum dos ergonomistas e fisioterapeutas do trabalho}

As LER/DORT são multifatoriais, de forma que fatores ambientais, biomecânicos, e pessoais interagem para determinar o risco de LER/DORT (Figura 1).

Estima-se que entre $11 \%$ e $95 \%$ das LER/ DORT podem ser prevenidas ao modificar as demandas físicas do trabalho ${ }^{15}$. Portanto, pesquisa em prevenção das LER/DORT é uma medida efetiva e fundamental ${ }^{16}$. As áreas de reabilitação e ergonomia possuem filosofias e objetivos similares $^{8}$. Os esforços preventivos (principalmente em países em desenvolvimento) deveriam ser de natureza participativa, envolvendo grupos multidisciplinares, e utilizando uma metodologia "de baixo para cima", começando pelos trabalhadores ${ }^{5}$. 


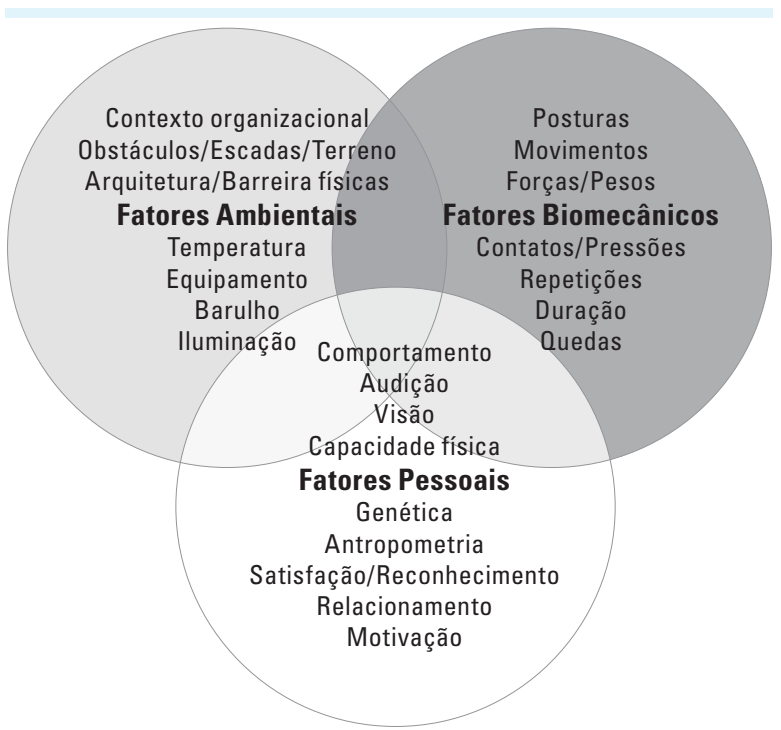

Figura l: Fatores interativos que afetam o risco de LER/DORT e alguns exemplos de possíveis mediadores do risco

Quando as demandas físicas do trabalho excedem as capacidades dos trabalhadores, o risco de LER/DORT aumenta significativamen$\mathrm{te}^{17}$. A prevenção e reabilitação destas desordens envolve a análise das demandas físicas do trabalho (ADF) e a avaliação das capacidades funcionais dos trabalhadores/pacientes (ACF). Os objetivos da ADF são quantificar o esforço físico imposto pelo trabalho em relação às posturas, movimentos, repetições, e duração das tarefas ocupacionais. Em relação à $\mathrm{ACF}$, seus objetivos são determinar o nível de performance que os trabalhadores/pacientes são capazes de trabalhar com segurança em relação às amplitudes de movimento utilizadas, força realizada, resistência, cinemática, cinética, propriocepção e coordenação. As informações coletadas com a ACF podem ser utilizadas para avaliar se os sujeitos estão aptos para o trabalho tanto na admissão inicial quanto no processo de retorno ao trabalho após uma lesão. O estudo dos pacientes/trabalhadores e trabalhos deve ser sistemático e poderia utilizar percentuais do movimento, resistência, e força normal, máxima e requerida pelo trabalho ${ }^{18}$. As relações entre os requesitos do trabalho e as capacidades dos trabalhadores são exploradas mais profundamente nos artigos de Kumar ${ }^{8}, 10$ incluindo as dimensões sociais e psicológicas além das demandas físicas do trabalho.

A ADF e a ACF devem ser consideradas em conjunto para melhores resultados ${ }^{19}$. Quando uma lesão relacionada ao trabalho ocorre (ou se possível antes), uma ADF deveria ser realizada. Além disso, o trabalhador lesado deveria passar por uma ACF com os devidos cuidados para não agravar a lesão. Os resultados podem ser utilizados para estudar a relação entre a lesão e as demandas do trabalho. Estes resultados devem ser utilizados para desenvolver o programa de reabilitação do trabalhador e para planejar modificações do trabalho a fim de prevenir outras lesões similares. Após o programa de reabilitação, uma segunda ACF deveria ser realizada para avaliar (I) se o trabalhador adquiriu ou restabeleceu o nível de capacidade funcional necessário ao seu trabalho; (II) se o trabalhador está apto para retornar ao trabalho; (III) se o trabalhador precisa de mais tempo de reabilitação e seus objetivos, ou (IV) se o trabalhador precisa mudar de atividade ocupacional e quais seriam os trabalhos que ele/ela poderia realizar.

Similarmente, após as modificações no trabalho definidas pela ADF inicial forem realizadas, uma nova ADF deve ser realizada para avaliar (I) se as modificações foram suficientes para adequar as demandas físicas e reduzir o risco de novos casos e re-lesões, ou (II) se intervenções adicionais são necessárias e quais seriam elas. Em longo prazo este procedimento resultará em um sistema de vigilância eficaz e em um grenciamento proativo das LER/DORT. O diagrama na Figura 2 apresenta o modelo proposto.

\section{Considerações Finais}

A integração da reabilitação e prevenção das LER/DORT, como proposto no modelo apresentado, poderá contribuir para uma atuação holística na promoção da saúde músculoesquelética no trabalho. A seguinte mudança de perspectiva é necessária para reduzir a dicotomia entre os profissionais e disciplinas. Os profissio- 


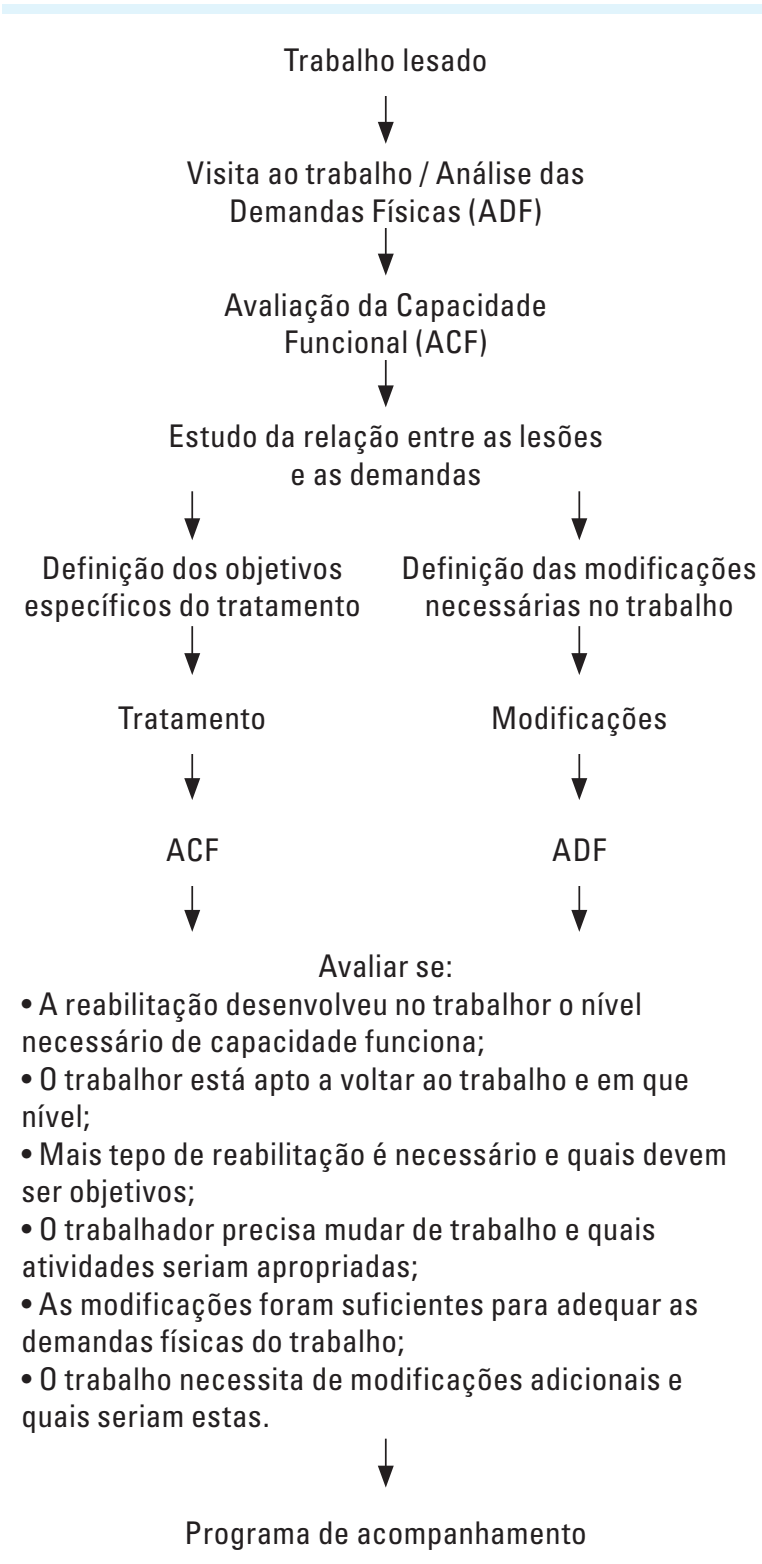

Figura 2: Modelo integrado de prevenção e reabilitação de LER/DORT

nais envolvidos na prevenção de lesões ocupacionais devem encarar a reabilitação das LER/ DORT como prevenção de incapacidade para o trabalho - prevenção secundária. Enquanto que os profissionais da reabilitação devem encarar a prevenção primária das LER/DORT como reabilitação com enfoque no ambiente e sistema de trabalho em vez de reabilitação com enfoque no paciente/trabalhador. Acredita-se que esta conduta resultará em maior sucesso no gerencia- mento das LER/DORT que atualmente afetam tantos trabalhadores e o sistema previdenciário.

\section{Āgradecimentos}

O modelo integrado de prevenção e reabilitação de LER/DORT apresentado neste artigo foi desenvolvido como parte de minha tese de doutorado: Work-Related Low Back Disorders in Heavy Jobs and their Control, 2006. 258f. Tese de Doutorado (PhD in Rehabilitation Science) Faculty of Rehabilitation Medicine, University of Alberta, Edmonton, Canadá, 2006. Esta pesquisa foi subvencionada pela Coordenação de Pessoal de Nível Superior - CAPES (bolsa de doutorado integral no exterior, BEX proc. n. 1340-01/8), e contou com o auxílio financeiro para pesquisa do Caritas Health Group e do Alberta CIHR Training Program in Bone and Joint Health (Canadian Institutes of Health Researc

\section{Referências}

1. World Health Organization. International Classification of Impairments, Disabilities and Handicaps 2001. 2ed. Disponível em: http://www3. who.int/icf/intros/ICF-Eng-Intro.pdf.

2. Bureau of Labor Statistics. Lost-Worktime Injuries and Illnesses: characteristics and resulting days away from work. 2001. Disponível em: http://www. bls.gov/iif/oshwc/osh/case/ostb1177.pdf.

3. Jucá R, Costa H, Berzoini R. Texto base da Minuta de Política Nacional de Segurança e Saúde do Trabalho. Imprensa Oficial 05/05/2005; (85).

4. Ministério da Saúde. LER/DORT afeta 310 mil trabalhadores na cidade de São Paulo. Folha de São Paulo, São Paulo, 07 de out. 2001, Caderno 9, p. 9.

5. O'neill DH. Ergonomics in industrially developing countries: does its application differ from that in industrially advanced countries? Appl Ergon. 2000; 31:631-640.

6. Wisner A. In praise of Brazilian ergonomics. Int J Ind Ergon. 1998; 21(5):415-419. 
7. Coury HJCG. Time trends in ergonomic intervention research for improved musculoskeletal health and comfort in Latin America. Appl Ergon. 2005; 36(2):249-252.

8. Kumar S. Rehabilitation and Ergonomics: Complementary Disciplines. Can J Rehab. 1989; 3(2):99-111.

9. Kumar S. Preface. Int J Ind Ergon. 1996; 17(2):77-79.

10. Kumar S. Rehabilitation: an ergonomic dimension. Int J Ind Ergon. 1992; 9(2):97-108.

11. Costa Bda, Vieira ER. Stretching to reduce workrelated musculoskeletal disorders: a systematic review. J Rehabil Med. 2008; 40:321-328. Disponível em: http://jrm.medicaljournals.se/article/ pdf/10.2340/16501977-0204.

12. American Physical Therapy Association. Occupational Health Physical Therapy Guidelines: the physical therapist in occupational health. 2005a. Disponível em: http://www.apta.org/Publications/ occ_health/role_of_pt.

13. American Physical Therapy Association. Occupational Health Physical Therapy Guidelines: Work-Related Injury/Illness Prevention and Ergonomics; 2005b. Disponível em: http://www.apta.org/Publications/ occ_health/workinjury_ergonomics.
14. Rossi CN. A arte da fisioterapia do trabalho no Brasil. SAUT. 2005; (2).

15. Punnett L, Wegman DH. Work-related musculoskeletal disorders: the epidemiologic evidence and the debate: State of the art research perspectives on musculoskeletal disorder causation and control. J Electromyogr Kinesiol. 2004; 14(1):13-23.

16. Kumar S. Preventive research - an effective therapy. In: Mital A, Karwowski W, editors. Ergonomics in Rehabilitation. London: Taylor and Francis; 1988. p. 183-197.

17. Vieira ER, Kumar S. Esforço físico ocupacional e saúde músculo-esquelética. Anais do XIII Congresso Brasileiro de Ergonomia; Outubro 2004; Fortaleza, Ceará, Brasil. CD-Room; 2004. Disponível em: http:// www.sportplusnet.com.br/arq/artigo.pdf

18. Vieira ER, Kumar S. Occupational biomechanical demand evaluation. Proceedings of the 2nd Annual Regional National Occupational Research Agenda; Abril 2004; University of Utah, Salt Lake City, Utah, USA. 2004; p. 171-80. Disponível em: http://www. mech.utah.edu/ergo/nora/2004/171-180_VieraEdgar. pdf.

19. Vieira ER. Work Physical Therapy and Rehabilitation Ergonomics: a review and discussion of the scope of the areas. Disabil Rehabil. 2006; 28(24):1563-1566. 
